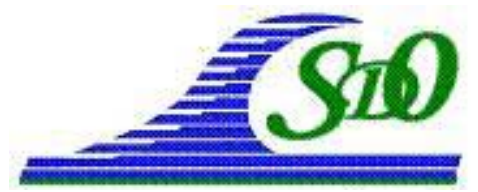

XI ${ }^{\text {èmes }}$ Journées Nationales Génie Côtier - Génie Civil

Les Sables d'Olonne, 22-25 juin 2010

DOI:10.5150/jngcgc.2010.073-P @ Editions Paralia CFL

disponible en ligne - http://www.paralia.fr - available online

\title{
Caractérisation des niveaux marins et modélisation des surcotes pendant la tempête Xynthia
}

\section{Lucia PINEAU-GUILLOU ${ }^{1}$, Cyril LATHUILIERE ${ }^{1}$, Rudy MAGNE ${ }^{1}$, Stéphanie LOUAZEL ${ }^{1}$, David CORMAN ${ }^{1}$, Céline PERHERIN ${ }^{2}$}

1. Service hydrographique et océanographique de la Marine,

13 rue du Chatellier, CS92803, 29228 Brest Cedex 2, France.

lucia.pineau-guillou@shom.fr; cyril.lathuiliere@shom.fr;rudy.magne@shom.fr ;

stephanie.louazel@shom.fr; david.corman@shom.fr

2. Centre d'Etudes Techniques Maritimes Et Fluviales,

Technopôle Brest Iroise, BP5, F-28280 Plouzané, France.

Celine.Perherin@developpement-durable.gouv.fr

\section{Résumé :}

Dans la nuit du 27 au 28 février 2010, la tempête Xynthia a traversé la France, engendrant des dégâts considérables. Les observations de niveau de la mer et de houle disponibles au SHOM ont été analysées pour caractériser l'événement. L'analyse des mesures marégraphiques, ainsi que l'exploitation des niveaux extrêmes calculés statistiquement, ont permis d'estimer les périodes de retour des niveaux marins atteints pendant cet événement, sur l'ensemble des côtes de la Manche et de l'Atlantique.

Les surcotes à La Rochelle ont été modélisées en prenant en compte les phénomènes la générant (pression atmosphérique, vent et vagues). L'élévation du niveau moyen due aux vagues ou "wave set-up" a ainsi été modélisée et prise en compte. Les simulations ont été réalisées à partir des codes WAVEWATCH III et HYCOM. Les surcotes modélisées du 20 février au $1^{\mathrm{er}}$ mars 2010 à La Rochelle ont été comparées aux surcotes mesurées par le marégraphe sur la même période, pour évaluer leur précision.

Mots-clés :

Hydrodynamique côtière - Niveaux marins extrêmes - Modélisation des surcotes Risques littoraux - État de mer - Tempête Xynthia

\section{Introduction}

La tempête Xynthia a traversé la France la nuit du 27 au 28 février 2010, engendrant la submersion marine exceptionnelle de certaines zones côtières. L'objectif de cette étude est d'une part d'estimer les périodes de retour des niveaux marins observés sur les côtes de la Manche et de l'Atlantique pendant cet événement, et d'autre part de modéliser les surcotes autour de La Rochelle en prenant en compte le "wave set-up" et de les comparer aux mesures disponibles pour évaluer leur précision. 


\section{Analyse des observations}

\subsection{Analyse des observations marégraphiques}

Les hauteurs d'eau acquises par les 18 marégraphes du Réseau d'Observation du Niveau de la Mer (RONIM) en Manche et Atlantique ont été analysées. La tempête a généré des surcotes importantes. La concomitance entre la tempête, l'instant de la pleine mer, et une vive-eau de coefficient de marée 102, explique que les niveaux atteints soient aussi importants. Les hauteurs de pleine mer ainsi que les surcotes de pleine mer mesurées (différence entre la hauteur de pleine mer observée et la hauteur de pleine mer prédite) sont synthétisées dans le tableau 1.

A la Rochelle, la surcote de pleine mer $(1,53 \mathrm{~m})$ est particulièrement importante (figure 1). Cette surcote n'a jamais été observée depuis l'installation du marégraphe (1997). Elle est supérieure à la surcote de pleine mer maximale observée à Brest (142 cm, enregistrée lors de la tempête du 15 octobre 1987), où l'on dispose de plus de 150 ans de mesures. Le niveau maximum atteint est de 8,01 m par rapport au zéro hydrographique (soit 4,51 m NGF), il n'avait également jamais été observé depuis l'installation de ce marégraphe. A titre de comparaison, le niveau atteint à La Rochelle lors de la tempête de 1999 était de 6,76 m par rapport au zéro hydrographique (soit 3,26 m NGF). Les périodes de retour ont été estimées à partir des statistiques de niveaux extrêmes. Pour 13 marégraphes, ces statistiques sont issues de l'analyse des graphes du produit commun SHOM-CETMEF (SIMON, 2008). Pour 4 des marégraphes ne figurant pas dans cette étude (Concarneau, Saint-Nazaire, Arcachon et Bayonne), elles ont été calculées. Enfin, pour le marégraphe du Crouesty, aucun calcul n'a pu être fait, car la durée des enregistrements est trop courte (marégraphe installé en 2002).

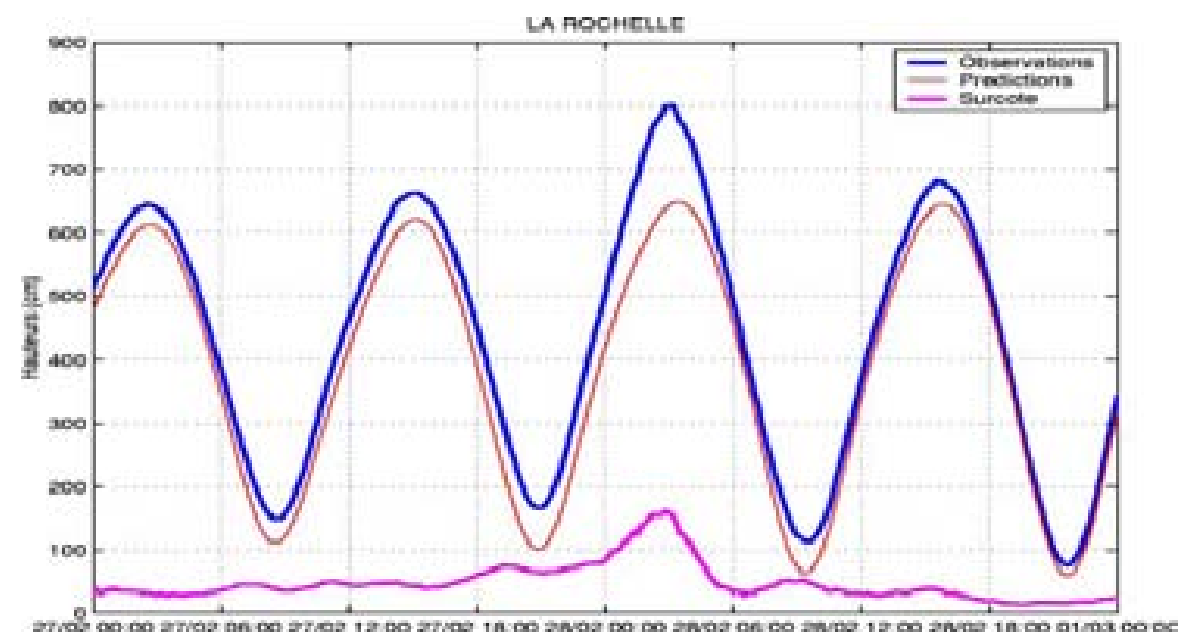

Figure 1. Hauteurs d'eau observées, hauteurs d'eau prédites, et surcotes instantanées à

La Rochelle, lors du passage de la tempête Xynthia. Les hauteurs prédites sont les valeurs officielles du SHOM évaluées par la méthode d'analyse harmonique. 

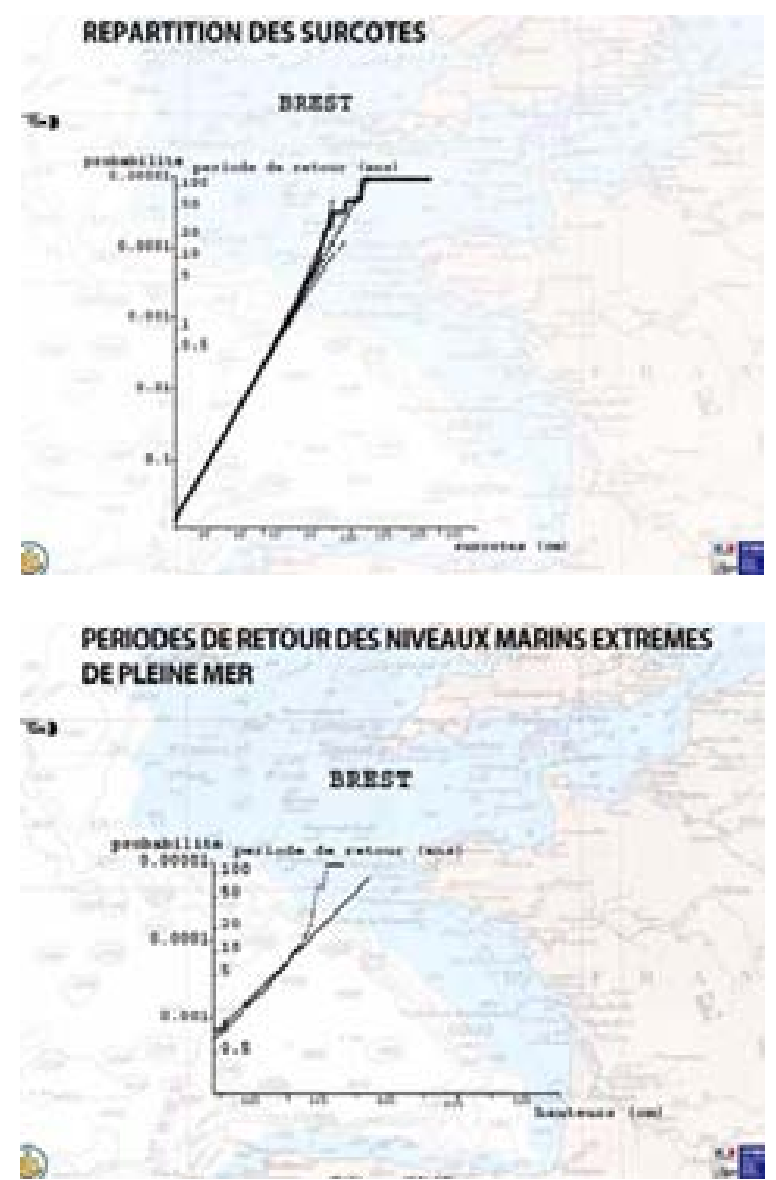

Figure 2. Répartition des surcotes et périodes de retour des niveaux extrêmes de pleine mer à Brest (SIMON, 2008).

L'estimation des périodes de retour est délicate au-delà de 100 ans. En effet, comme présenté sur l'exemple de la figure 2 du graphe de répartition des surcotes à Brest, quelques événements extrêmes correspondent à des événements atypiques, appelés "horsains" dont la valeur s'éloigne de la courbe issue de l'analyse statistique des données disponibles sur le site d'observation. Le terme "horsain" désigne une donnée qui se distingue par sa valeur significativement différente de celles des autres données de l'échantillon concerné. La prise en compte des horsains nécessite des études complémentaires, encore à mener, pour définir l'approche statistique la plus adaptée. Aussi, il n'a volontairement pas été estimé de valeur numérique précise de la période de retour lorsque que celle-ci est estimée supérieure à 100 ans.

Le tableau 1 et la figure 3 synthétisent les estimations des périodes de retour associées aux surcotes et aux hauteurs de pleine mer observées.

La zone la plus touchée s'étend de St-Nazaire à La Rochelle, avec des périodes de retour de hauteur de pleine mer supérieures à 100 ans. Les sites de la Pointe de Grave et Arcachon ont également enregistré des hauteurs de pleine mer exceptionnelles, de périodes de retour comprises entre 20 et 50 ans. 
Tableau 1. Périodes de retour associées aux surcotes et aux hauteurs de pleine mer observées (les hauteurs sont rapportées au zéro hydrographique).

\begin{tabular}{|c|c|c|c|c|c|c|}
\hline Marégraphe & $\begin{array}{l}\text { Surcote de } \\
\text { pleine mer } \\
\text { observée }\end{array}$ & $\begin{array}{l}\text { Période de } \\
\text { retour } \\
\text { associée à la } \\
\text { surcote de } \\
\text { pleine mer } \\
\text { observée }\end{array}$ & $\begin{array}{l}\text { Hauteur de } \\
\text { pleine mer } \\
\text { observée }\end{array}$ & $\begin{array}{l}\text { Période de } \\
\text { retour } \\
\text { associée à la } \\
\text { hauteur de } \\
\text { pleine mer } \\
\text { observée }\end{array}$ & $\begin{array}{l}\text { Niveau des } \\
\text { plus hautes } \\
\text { mers astro- } \\
\text { nomiques }\end{array}$ & $\begin{array}{l}\text { Cote du zéro } \\
\text { IGN69 par } \\
\text { rapport au } \\
\text { zéro hydro- } \\
\text { graphique }\end{array}$ \\
\hline Dunkerque & $0,80 \mathrm{~m}$ & $\sim 1$ an & $6,90 \mathrm{~m}$ & $1 \grave{a} 5$ ans & $6,48 m$ & $2,693 m$ \\
\hline Calais & $0,70 \mathrm{~m}$ & $1 \grave{a} 5$ ans & $8,05 \mathrm{~m}$ & $1 \grave{a} 5$ ans & $7,86 \mathrm{~m}$ & $3,459 \mathrm{~m}$ \\
\hline Dieppe & $1,05 \mathrm{~m}$ & 20 à 50 ans & $10,47 \mathrm{~m}$ & $5 \grave{a} 10$ ans & $10,11 \mathrm{~m}$ & $4,448 m$ \\
\hline Le Havre & $0,87 \mathrm{~m}$ & 1 à 5 ans & $8,84 m$ & 1 à 5 ans & $8,42 m$ & $4,378 m$ \\
\hline Cherbourg & $0,57 \mathrm{~m}$ & 1 à 5 ans & $7,14 m$ & $1 \grave{a} 5$ ans & $7,04 m$ & $3,285 \mathrm{~m}$ \\
\hline Saint-Malo & $0,88 \mathrm{~m}$ & $\sim 20$ ans & $13,18 \mathrm{~m}$ & $<1$ an & $13,47 \mathrm{~m}$ & $6,289 \mathrm{~m}$ \\
\hline Roscoff & $0,53 \mathrm{~m}$ & $<1$ an & $9,63 \mathrm{~m}$ & $<1$ an & $9,76 \mathrm{~m}$ & $4,764 m$ \\
\hline Le Conquet & $0,51 \mathrm{~m}$ & $<1$ an & $7,63 m$ & $<1$ an & $7,69 m$ & $3,502 \mathrm{~m}$ \\
\hline Brest & $0,53 \mathrm{~m}$ & $<1$ an & $7,89 \mathrm{~m}$ & $<1$ an & 7,93 m & $3,636 \mathrm{~m}$ \\
\hline Concarneau & $0,63 \mathrm{~m}$ & $5 \grave{a} 10$ ans & $5,90 \mathrm{~m}$ & $<1$ an & $5,63 \mathrm{~m}$ & $2,534 \mathrm{~m}$ \\
\hline Le Crouesty & $0,89 \mathrm{~m}$ & Non calculée & $6,58 \mathrm{~m}$ & Non calculée & $6,01 \mathrm{~m}$ & $2,850 \mathrm{~m}$ \\
\hline Saint-Nazaire & $1,16 \mathrm{~m}$ & 20 à 50 ans & $7,34 m$ & $>100$ ans & $6,59 \mathrm{~m}$ & $3,159 \mathrm{~m}$ \\
\hline Sables d'Olonne & / & I & $>6,89 m$ & $>100$ ans & $5,93 \mathrm{~m}$ & $2,831 \mathrm{~m}$ \\
\hline La Rochelle & $1,53 \mathrm{~m}$ & $>100$ ans & $8,01 \mathrm{~m}$ & $>100$ ans & $6,86 \mathrm{~m}$ & $3,504 \mathrm{~m}$ \\
\hline Pointe de Grave & $0,98 \mathrm{~m}$ & 10 à 20 ans & $6,57 \mathrm{~m}$ & $20 \grave{a} 50$ ans & $5,96 \mathrm{~m}$ & $2,831 \mathrm{~m}$ \\
\hline Arcachon & $0,90 \mathrm{~m}$ & 5 à 10 ans & $5,46 m$ & 20 à 50 ans & $4,86 m$ & $1,980 \mathrm{~m}$ \\
\hline Bayonne & $0,38 \mathrm{~m}$ & $<1$ an & $4,92 \mathrm{~m}$ & $<1$ an & $4,84 m$ & $2,143 \mathrm{~m}$ \\
\hline St Jean de Luz. & $0,38 \mathrm{~m}$ & $<1$ an & $4,96 \mathrm{~m}$ & $<1$ an & $4,90 \mathrm{~m}$ & $2,175 \mathrm{~m}$ \\
\hline
\end{tabular}

\subsection{Analyse de l'état de mer}

Depuis le 2 janvier 2010, le SHOM mesure la houle aux abords de l'île d'Oléron $\left(45^{\circ} 50,5^{\prime} \mathrm{N}-01^{\circ} 48,8^{\prime} \mathrm{W}\right)$ au moyen d'une bouée directionnelle (figure 4).

L'événement est caractérisé par une évolution très rapide de la houle. En trois heures, la hauteur des vagues passe de moins de $3 \mathrm{~m}$ à 7,5 $\mathrm{m}$ (figure 5). La direction et la période (figure 5) évoluent également très vite, passant respectivement du Sud au Ouest-SudOuest et de $5 \mathrm{~s}$ à $9 \mathrm{~s}$ sur ces 3 heures. Le maximum d'énergie est atteint entre $03 \mathrm{~h} 00$ et 06h00 UT avec des hauteurs significatives supérieures à $7 \mathrm{~m}$ et des périodes supérieures à $8 \mathrm{~s}$.

A titre de comparaison, les hauteurs observées dans la nuit du 27 au 28 février 2010 sont du même ordre de grandeur que celles prévues par les modèles de vagues du SHOM pendant les tempêtes de décembre 2007 ou mars 2008. 


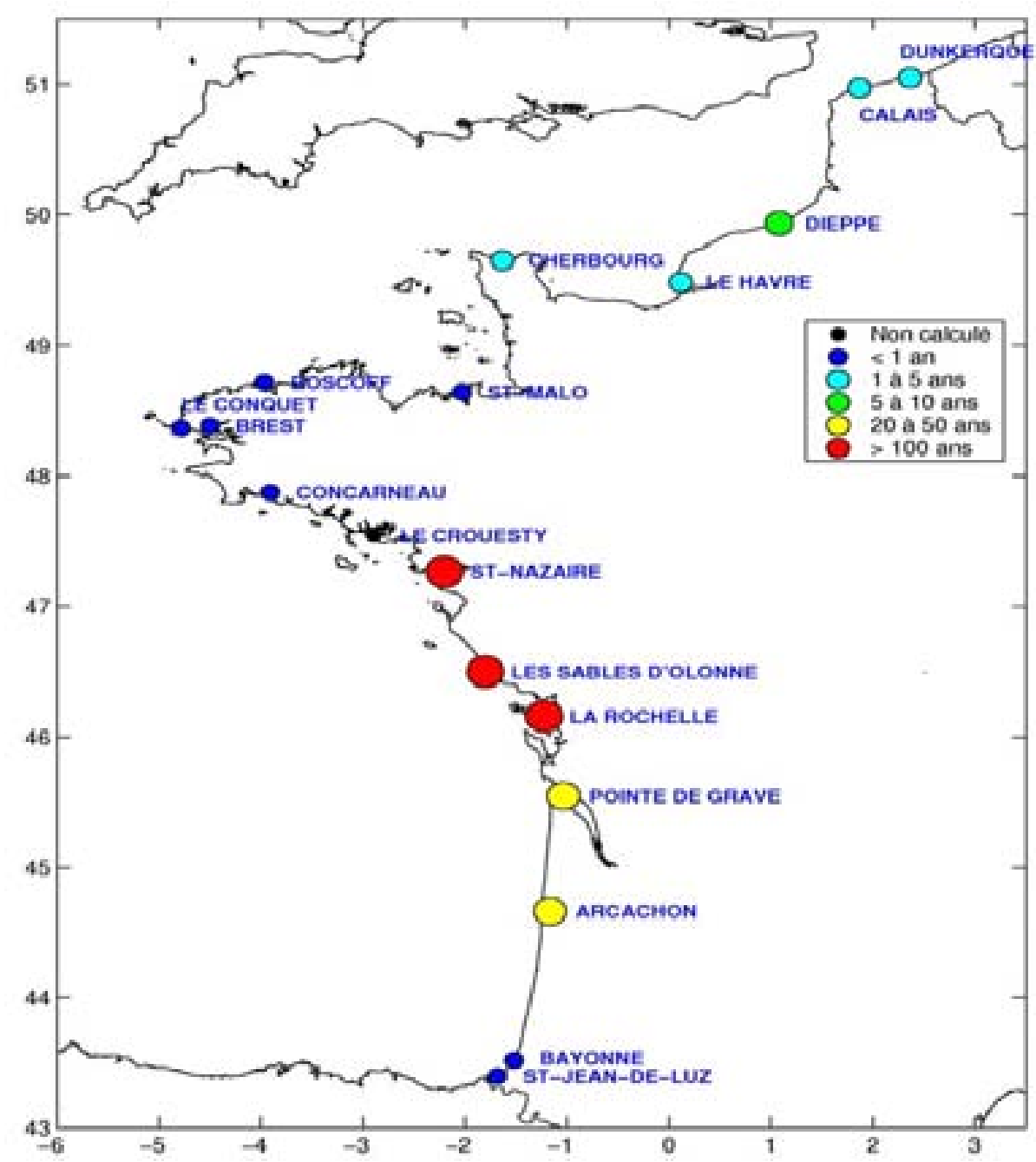

Figure 3. Estimation des périodes de retour associées aux hauteurs de pleine mer observées lors du passage de la tempête Xynthia.

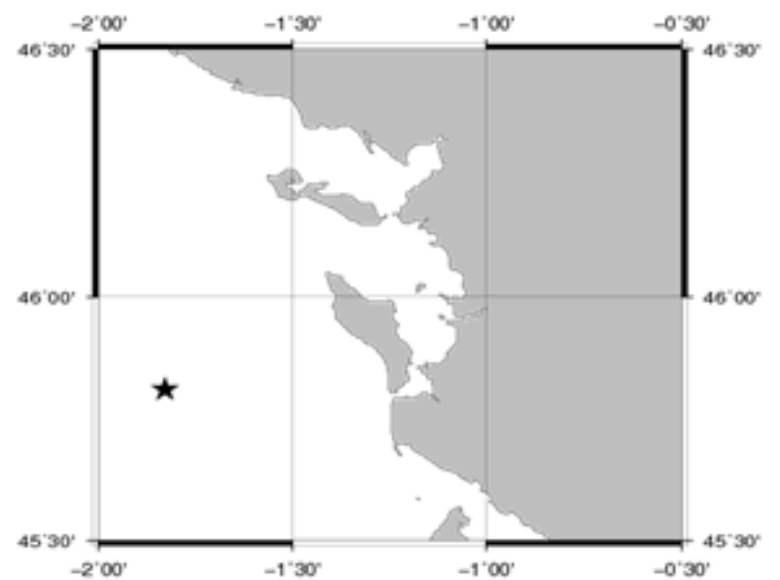

Figure 4. Situation de la bouée directionnelle du SHOM (étoile). 

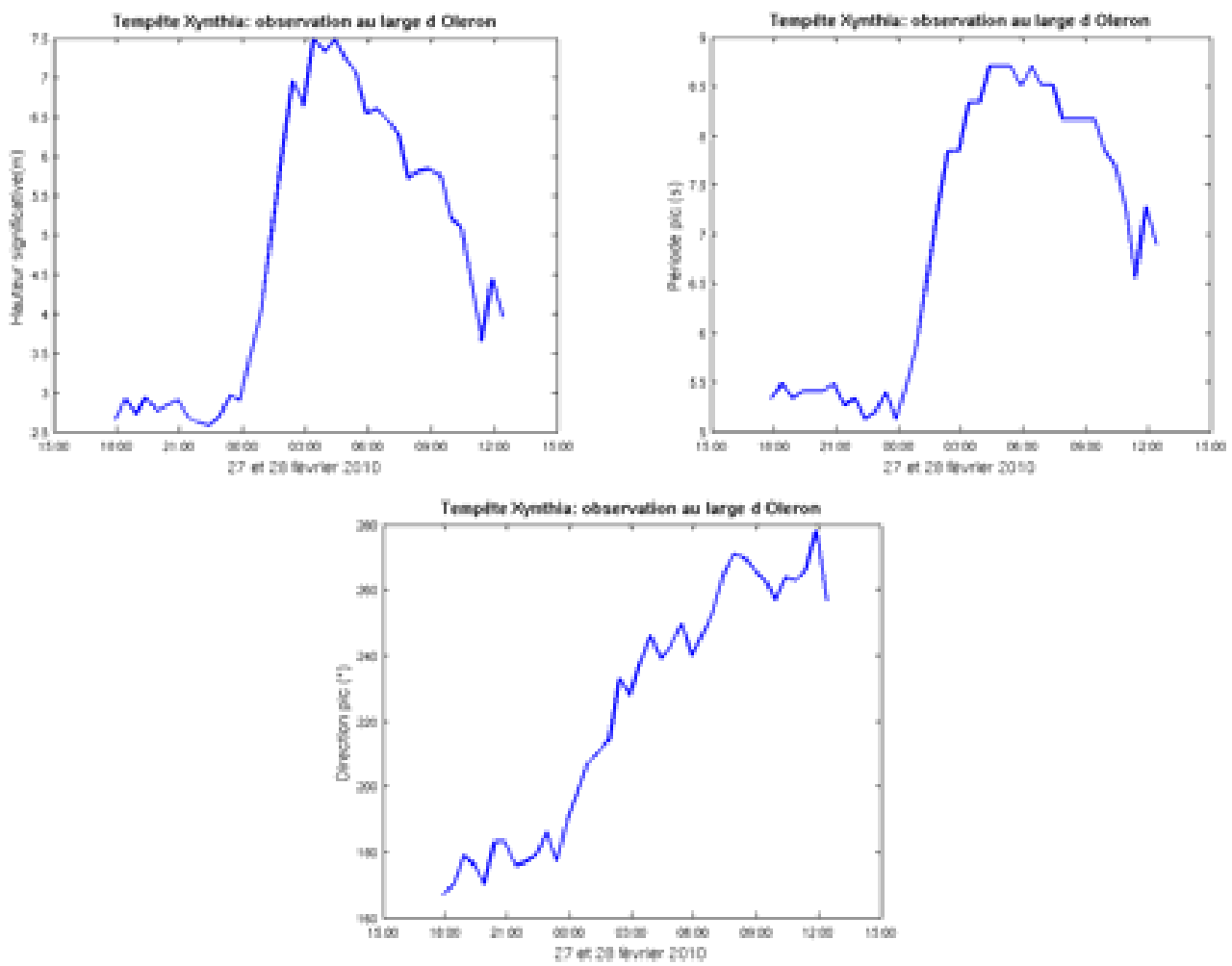

Figure 5. Hauteur significative, période et direction pic de la houle mesurées au large d'Oléron pendant la tempête Xynthia.

\section{Modélisation de l'hydrodynamique marine pendant la tempête Xynthia}

\subsection{Modèle de circulation océanique HYCOM}

Le modèle de circulation océanique HYCOM est développé en partie par le SHOM (MOREL et al., 2008), membre du consortium de développement, piloté par l'Université de Miami. Le modèle de la Manche et du Golfe de Gascogne, de résolution spatiale $3 \mathrm{~km}$, s'étend de $15^{\circ} \mathrm{W}$ à $2^{\circ} \mathrm{E}$ et de $43^{\circ} \mathrm{N}$ à $51^{\circ} \mathrm{N}$. Dans les résultats présentés, les données de vent, fournies par Météo France, sont issues du Centre Européen de Prévision (CEP) et intégrées avec une résolution temporelle de 3 heures et une résolution spatiale de $0,25^{\circ}$.

L'effet des vagues et de la pression atmosphérique sur les niveaux de la mer n'est pas inclus dans HYCOM. Une simulation HYCOM a été réalisée sans prendre en compte la marée, elle comprend donc les effets de la tension de vent sur l'océan ainsi que les variations éventuelles de la hauteur d'eau dues à la circulation générale de l'océan (tourbillons, ondes côtières, etc, ...). Le processus dominant étant le vent, cette surcote est appelée par la suite "surcote due au vent" (figure 6). Elle atteint environ $50 \mathrm{~cm}$ au niveau de la Vendée à 03 h00 UT du matin le 28 février. 


\section{XI ìmes Journées Nationales Génie Côtier-Génie Civil}

Les Sables d'Olonne, 22-25 juin 2010

\subsection{Modèle de vagues WAVEWATCH III}

Le modèle de vagues WAVEWATCH III est un modèle développé par la NOAA (TOLMAN, 2009). Le SHOM participe aux évolutions, développements et validations de ce modèle en collaboration avec la NOAA et l'IFREMER.

Le modèle WAVEWATCH III permet de calculer l'évolution de l'énergie de la houle sur un domaine. A partir de ces paramètres, il est possible de calculer une surélévation du niveau moyen due aux vagues ("wave set-up"). Les calculs présentés correspondent au maximum géographique des surcotes (à la côte après le déferlement). Cependant, ces calculs ne prennent pas en compte le niveau maximal instantané ("run-up") qui est lié au déferlement individuel de chaque vague et très dépendant de la pente locale de plage.

La configuration utilisée (figure 7) est un modèle à 2 minutes de résolution sur la façade Atlantique Française avec des forçages (vent de surface) du Centre Européen de Prévision à $0,25^{\circ}$ de résolution spatiale et 3 heures de résolution temporelle. Les données modélisées (vagues) issues de ce domaine ont pu être validées grâce aux observations au large d'Oléron (figure 5).

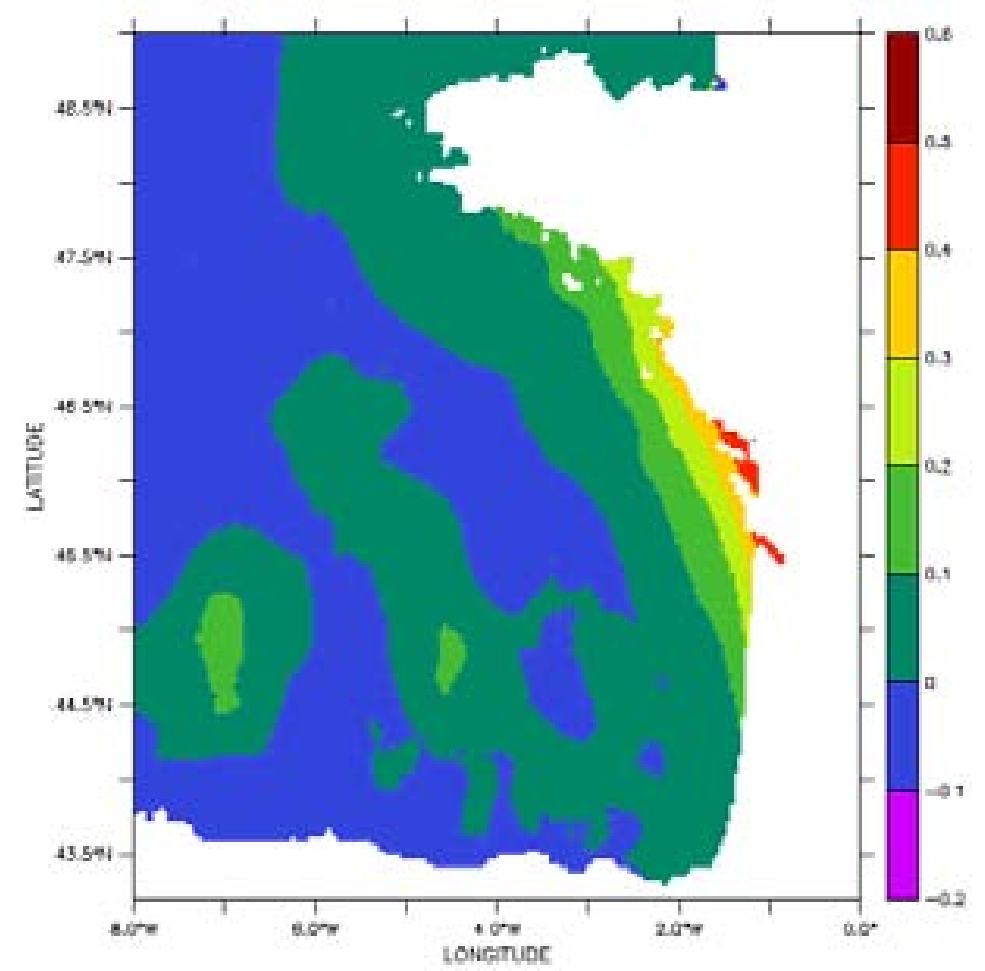

Figure 6. Surcote due au vent issue de HYCOM le 28 février à $2 \mathrm{~h} U T$.

La figure 8 présente les prévisions de surcotes dues aux vagues (courbe rouge) associées aux hauteurs de vagues déferlantes (courbe bleue) devant La Rochelle entre mi et fin février 2010. 


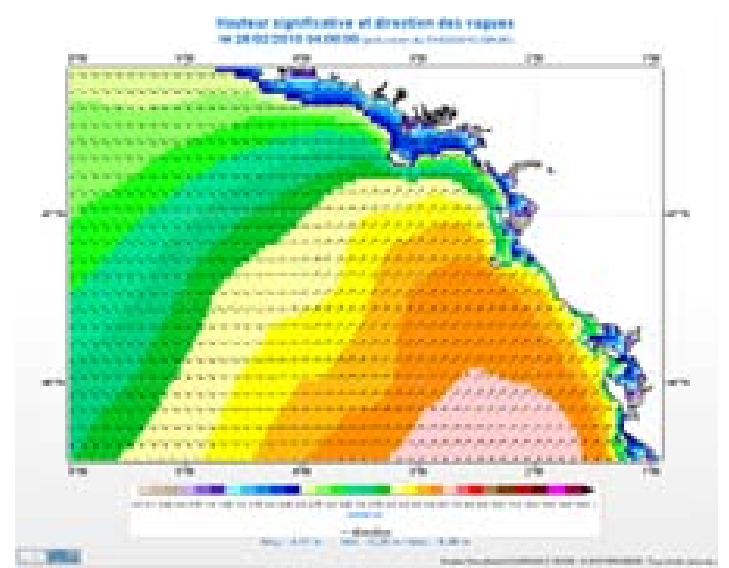

Figure 7. Hauteurs et directions des vagues le 28 février à 4 h UT+1 autour de La Rochelle (PREVIMER, site web).

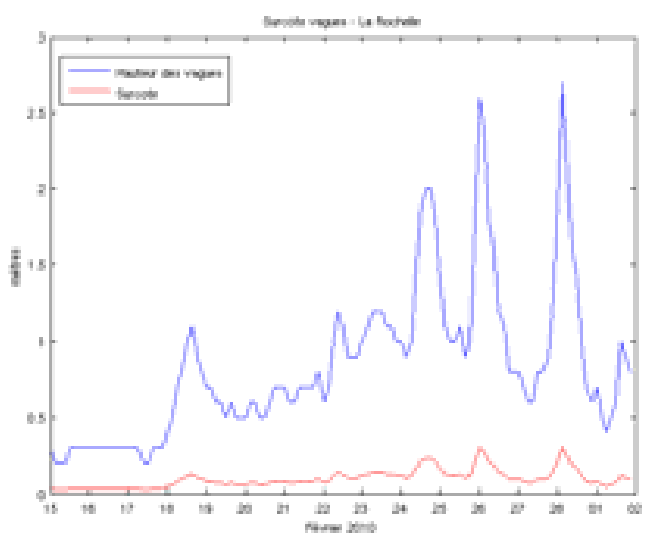

Figure 8. Hauteur des vagues et surcote due aux vagues (wave set-up) à La Rochelle en février 2010.

\subsection{Modélisation des surcotes totales}

Les différentes contributions élémentaires aux surcotes totales ont été calculées à La Rochelle :

- surcote due à la pression atmosphérique, qui est évaluée à partir de la correction dite de "baromètre inverse" ;

- surcote due au vent, qui est calculée à partir de la simulation HYCOM ;

- surcote due aux vagues, qui est calculée à partir de WAVEWATCH III.

La surcote totale modélisée a été comparée à la surcote mesurée au niveau du marégraphe de la Rochelle. Les résultats obtenus (figure 9) sont encourageants, il n'existe pas de biais significatif entre les courbes. La haute fréquence au niveau des mesures vient du fait qu'elles sont acquises toutes les 10 minutes, alors que pour le modèle, les données de vent et le "wave set-up" ne sont obtenus que toutes les 3 heures. L'ensemble des événements est bien représenté. Cependant, le "pic" du 28 février est sous-estimé, avec une surcote instantanée maximale de $1,3 \mathrm{~m}$ alors que la valeur mesurée atteint 1,6 $\mathrm{m}$. Ceci pourrait probablement être amélioré en augmentant la fréquence des forçages du vent ( 3 heures actuellement) et la résolution spatiale $(3 \mathrm{~km}$ pour cette maquette).

Une étude de sensibilité a permis de mettre en évidence l'impact significatif de la résolution spatio-temporelle des données de vent sur l'amplitude de la surcote (surcote due au vent de $25 \mathrm{~cm}$ au lieu de $65 \mathrm{~cm}$, en cas de dégradation de la résolution spatiotemporelle). 


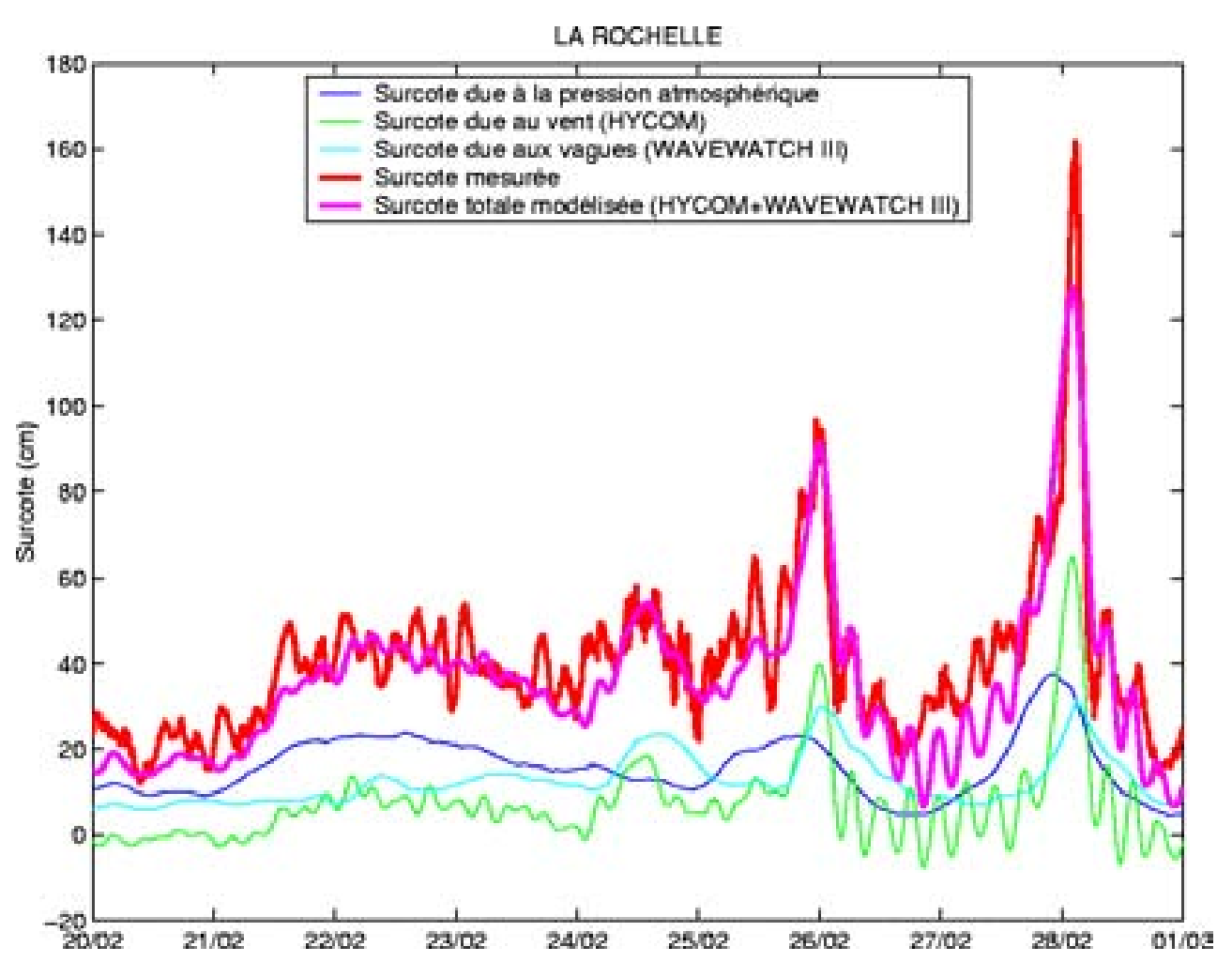

Figure 9. Surcotes modélisées et mesurées au niveau de La Rochelle en février 2010.

\section{Conclusion}

L'analyse des données SHOM a permis d'estimer les périodes de retour associées aux niveaux de pleine mer observés pendant la tempête Xynthia sur l'ensemble des côtes de la Manche et de l'Atlantique. Les niveaux de pleine mer extrêmes observés entre SaintNazaire et La Rochelle, de période de retour supérieure à 100 ans, sont dus à la concomitance entre la tempête, l'instant de la pleine mer, et une marée de vive-eau de coefficient important.

La modélisation de la tempête a permis d'obtenir des surcotes de précision satisfaisante grâce à l'exploitation conjointe des modèles HYCOM et WAVEWATCH III. Les tempêtes étant des phénomènes aléatoires non prédictibles, la modélisation reste un outil incontournable pour permettre la prévision.

\section{Références bibliographiques}

MOREL Y., BARAILLE R., PICHON A. (2008). Time splitting and linear stability of the slow part of the barotropic component. Ocean Modelling, Vol. 23, pp 73-81. doi:10.1016/j.ocemod.2008.04.001

PREVIMER (site web). http://www.previmer.org/

SIMON B. (2008). Statistiques des niveaux marins extrêmes de pleine mer en Manche et Atlantique. CD-Rom, Produit commun SHOM-CETMEF. 
Thème 4 - Vulnérabilité du littoral

TOLMAN H.L. (2009). User manual and system documentation of WAVEWATCH III TM version 3.14. NOAA / NWS / NCEP / MMAB Technical Note *276*, $194 \mathrm{pp}+$ Appendices. Disponible en ligne sur :

URL http://polar.ncep.noaa.gov/mmab/papers/tn276/MMAB_276.pdf 